Open Access

Original Article

\title{
Emerging resistance of van genotype in enterococci: A potential menace for therapeutic failure
}

\author{
Hasan Ejaz
}

\begin{abstract}
Objectives: Emerging cases of vancomycin-resistant enterococci (VRE) are detrimental for the patients. The current study aimed to ascertain the occurrence of VRE, their antibiogram and the van genotype responsible for vancomycin resistance.

Methods: A total number of 2,958 clinical specimens were processed at Microbiology Department of the Alrazi Health Care, Lahore during the one year (2016-2017) using microbiological culture media, biochemical and serology. Antibiogram of enterococcal strains was performed using disc diffusion and E-test. ATCC Enterococcus faecalis 29212 was used as a quality control strain. The detection of van genotypes was accomplished by multiplex PCR assay.

Results: Out of the 147 enterococci, 139 (94.6\%) were $E$. faecalis, and 8 (5.4\%) were $E$. faecium. Statistically significant associations of urine $(p<0.001)$, pus $(p<0.001)$ and wound swabs $(p=0.001)$ were observed with $E$. faecalis. A significant correlation of enterococcal infections $(p=0.05)$ was seen with female patients. Four $(2.9 \%)$ strains of $E$. faecalis found to be VRE with vanB $(75 \%)$ and vanA $(25 \%)$ genotypes.

Conclusion: The emerging strains of VRE ( $v a n B$ and vanA genotype) in the current study are a potential menace for therapeutic failure, which left the physicians with only linezolid as a therapeutic option.

KEYWORDS: E. faecalis, E. faecium, Glycopeptide resistance, Linezolid, Vancomycin resistant enterococci, van genotypes.
\end{abstract}

doi: https://doi.org/10.12669/pjms.35.6.1145

How to cite this:

Ejaz $H$. Emerging resistance of van genotype in enterococci: A potential menace for therapeutic failure. Pak J Med Sci. 2019;35(6):1659-1663. doi: https://doi.org/10.12669/pjms.35.6.1145

This is an Open Access article distributed under the terms of the Creative Commons Attribution License (http://creativecommons.org/licenses/by/3.0), which permits unrestricted use, distribution, and reproduction in any medium, provided the original work is properly cited.

\section{INTRODUCTION}

Enterococcus species were considered to be harmless and unimportant to humans medically many years ago. They have the ability to produce bacteriocins, probiotic and have been used extensively in the food industry. ${ }^{1}$ Medically, they are opportunistic, lactic acid Gram-positive cocci

1. Dr. Hasan Ejaz, PhD

Department of Clinical Laboratory Sciences,

CAMS, Jouf University,

Sakaka, Saudi Arabia.

Correspondence:

Dr. Hasan Ejaz

College of Applied Medical Sciences, Jouf University, King Khalid Road,

Sakaka, Saudi Arabia.

Email: hasanmicro@gmail.com

* Received for Publication:

* Revision Received:

* Revision Accepted:

May 26, 2019

August 26, 2019

August 28, 2019 which are mainly residents of gastrointestinal (GI) tract of humans and animals but now they have been contributed approximately $80-90 \%$ of human enterococcal infections in which $E$. faecalis and $E$. faecium play the major part. $^{2}$ They have emerged as a significant part of nosocomial pathogens among others and are a reason for increased rate of mortality. ${ }^{3}$ Multidrug-resistant enterococcal infections should be given the same significance as methicillin-resistant Staphylococcal infections and the infections caused by extended-spectrum betalactamase-producing bacterial strains. ${ }^{4-6}$

More than 17 species of genus Enterococcus have been discovered, but E. faecalis and E. faecium are the predominating. Some other species (E. avium, E. gallinarum and E. casseliflavus) are also accountable for infections in humans. ${ }^{7}$ Enterococci can cause a variety of infections which can be life-threating such as endocarditis bacteremia, intravenous catheter infection and UTI. ${ }^{8}$ They are multidrug 
resistance against extensive classes of drugs that include beta-lactams and aminoglycosides and have left only few treatment options for clinicians. The acquisition of resistance is accomplished by transposons, plasmids and chromosomal transfer or mutations against different groups of antibiotics. From the past two decades, enterococci have acquired resistance against many commonly used drugs and their potential for gaining and spreading resistant genes with ease is alarming. ${ }^{9}$

The vancomycin resistance amongst the enterococcal species identified in the 1980s in England. A pronounced increase has been found in the popularity of the vancomycin resistance, which is a glycopeptide antibiotic. ${ }^{10}$ Diverse types of vancomycin-resistant genes have been identified, which is worrisome and keep a significant concern. Enterococcal glycopeptide resistance can be mediated by the van $A$ (vancomycin and teicoplanin resistance), vanB genes (vancomycin resistance) and vanM (vancomycin and teicoplanin resistance) genes. ${ }^{11}$ The rest of the van genes responsible for the glycopeptide resistance are $\operatorname{van} C$, vanD, vanE, vanG, vanL and vanN. ${ }^{12}$ Prompt and precise detection of vancomycin-resistant enterococci (VRE) is necessary to reduce the morbidity among the infected patients which is usually done by the molecular methods. ${ }^{13}$ The current study aimed to ascertain the prevalence of VRE in various clinical sources, their antibiogram and genetic basis of vancomycin (van genotype) resistance.

\section{METHODS}

The bacterial strains for this cross-sectional study were collected from the Microbiology Department of the Alrazi Health Care Lahore, Pakistan, over twelve months from July 2016 to June 2017 after IRB approval (Ref.no. 13A/ARHC, Date on: June $15,2016)$. A total number of 2,958 various clinical samples of urine, pus, wound swabs, ear swabs, endotracheal tubes (ETT), blood, cerebrospinal fluid (CSF) and other body fluids were processed to detect the occurrence of enterococci consecutively. Samples were processed to isolate enterococci from both genders of different age groups.

The clinical specimens were inoculated on different culture media (Blood, Chocolate, MacConkey's and CLED) and incubated at $37^{\circ} \mathrm{C}$ for overnight. ${ }^{14}$ Enterococci were identified by physical characteristics, Gram's reaction, biochemical (catalase reaction) and serological testing (reaction to Group-D antisera). Moreover, the tolerance of enterococci was checked by $6.5 \% \mathrm{NaCl}$ and the ability to grow on bile esculin agar. The non-enterococcal concomitant organisms were excluded from the study.

Enterococcal antibiogram was performed by using Kirby Bauer disc diffusion method, and bacterial suspensions were made according to 0.5 McFarland's scale by streaking the bacterial suspension on the surface of the blood agar plate. The antibiotic discs of penicillin (10 $\mathrm{U})$, ampicillin $(10 \mu \mathrm{g})$, co-amoxiclav $(20 / 10 \mu \mathrm{g})$, ceftazidime $(30 \mu \mathrm{g})$, linezolid $(30 \mu \mathrm{g})$, ceftriaxone $(30 \mu \mathrm{g})$, cefuroxime $(30 \mu \mathrm{g})$, piperacillin-tazobactam $(100 / 10$ $\mu \mathrm{g})$, clindamycin $(10 \mu \mathrm{g})$ and erythromycin $(15 \mu \mathrm{g})$ were used to observe the antibiogram. E-test strips (Liofilchem, Italy) were used to determine the minimum inhibitory concentration of vancomycin. After 24 hours' incubation at $35-37^{\circ} \mathrm{C}$, results of these drugs were recorded as mentioned in the Clinical and Laboratory Standard Institute (CLSI) manual. ATCC E. faecalis 29212 was used as a quality control strain. Results of each isolate were reported as sensitive, intermediate sensitive or resistant to the antimicrobial disc based on the interpretation chart of zone sizes recommended by CLSI. ${ }^{15}$

The detection of van $A, \operatorname{van} B, \operatorname{van} C 1$ and $\operatorname{van} C 2 / C 3$ was done using multiplex PCR assay. The previously mentioned primers were used for the detection of van $A, \operatorname{vanB}$, van $\mathrm{C} 1$ and $\operatorname{van} \mathrm{C} 2 / \mathrm{C} 3 .{ }^{16}$ The DNA was extracted in $100 \mu \mathrm{TE}$ buffer using boiling method for 10 minutes. The PCR conditions were optimised using different gradient temperatures for annealing and finally adjusted as initial denaturation $\left(95^{\circ} \mathrm{C}\right.$, 10 minutes) followed by 29 cycles of denaturation $\left(95^{\circ} \mathrm{C}, 1\right.$ minute), annealing $\left(55^{\circ} \mathrm{C}, 50\right.$ seconds $)$, DNA extension $\left(72^{\circ} \mathrm{C}, 55\right.$ seconds) and final extension at $72^{\circ} \mathrm{C}$ for 10 minutes. Multiplex PCR products were analysed using 1\% Agarose gel, SYBR Safe (gel staining dye) and $100 \mathrm{bp}$ molecular ruler. No template control was also included with each run of electrophoresis. Chi-square test used to analyze the p-values for the statistical analysis using SPSS v.24.

\section{RESULTS}

A total of 147 enterococcal strains were isolated from 2,958 different clinical isolates, amongst which $139(94.6 \%)$ were E. faecalis and eight (5.4\%) were E. faecium. Primarily, the sources of both of the enterococci were urine, pus and wound swabs. Statistically significant associations of urine $(\mathrm{p}<$ $0.001)$, pus $(p<0.001)$ and wound swabs $(p=0.001)$ were observed with $E$. faecalis. No statistically significant relationship of E. faecalis or E. faecium found with the other sources. Overall, the frequency of $E$. faecalis was higher in female patients $(\mathrm{n}=92$; 
Emerging van genotype in enterococci

Table-I: Source, age and gender distribution of cases infected with enterococci $(n=147)$.

\begin{tabular}{lcccc}
\hline Specimens & Total cases $n(\%)$ & E. faecalis $n(\%)$ & E. faecium n $(\%)$ & $p$-value \\
\hline Urine & $1244(42.1)$ & $94(63.9)$ & $5(3.4)$ & $<0.001$ \\
Pus & $734(24.8)$ & $26(17.7)$ & $2(1.4)$ & $<0.001$ \\
Wound Swab & $275(9.3)$ & $13(8.8)$ & $1(0.7)$ & 0.001 \\
Ear Swab & $172(5.8)$ & $3(2)$ & 0 & - \\
ETT Tips & $19(0.6)$ & $2(1.4)$ & 0 & - \\
Blood & $452(15.3)$ & $1(0.7)$ & 0 & - \\
Other Body Fluids & $50(1.7)$ & 0 & 0 & - \\
CSF & $12(0.4)$ & 0 & 0 & - \\
\hline Total & $\mathrm{n}=2958$ & $139(94.6)$ & $8(5.4)$ & \\
\hline Gender [Enterococcal cases; $\boldsymbol{n}$ (\%)] & & & & \\
Males & $49(33.3)$ & $47(32)$ & $2(1.4)$ & 0.05 \\
Females & $98(66.7)$ & $92(62.5)$ & $6(4.1)$ & \\
Age Groups [Enterococcal cases; $\boldsymbol{n}(\%)]$ & & & & \\
Mean age \pm S.D. & $57.5 \pm 24.6$ & $54.2 \pm 16.5$ & 0.85 \\
0-20 years & $18(12.2)$ & $18(12.2)$ & $3(2)$ & \\
21-40 years & $60(40.8)$ & $57(38.8)$ & $5(3.4)$ & \\
41-60 years & $58(39.5)$ & $53(36.1)$ & 0 & \\
61-80 years & $11(7.5)$ & $11(7.5)$ & $8(5.4)$ & \\
\hline Total & 147 & $139(94.6)$ & & \\
\hline
\end{tabular}

$62.5 \%)$ than male patients $(n=47 ; 32 \%)$. Similarly, the frequency the E. faecium was higher among the female patients $(\mathrm{n}=6 ; 4.1 \%)$ than male patients $(n=2 ; 1.4 \%)$. A borderline significant correlation of enterococcal infections $(p=0.05)$ was seen with female patients. The correlation of the patients with different age groups in the case of $E$. faecalis and $E$. faecium infections was not statistically significant $(\mathrm{p}=0.85)$. The mean patient age \pm SD in case if $E$. faecalis was $57.5 \pm 24.6$ while the mean age $\pm S D$ in case of E. faecium was $54.2 \pm 16.5$ (Table-I).

In vitro antibiogram of enterococci was observed against the various groups of antibiotics. All the isolated enterococcal strains were resistant to penicillin. There were 109 (78.4\%) strains E. faecalis resistant to ampicillin, $99(71.2 \%)$ to ceftriaxone, $98(70.5 \%)$ to piperacillin-tazobactam, $90(64.7 \%)$ to cefuroxime and 75 (54\%) to ciprofloxacin. There were four $(2.9 \%)$ strains E. faecalis found to be resistant to vancomycin. The resistance profile of $E$. faecium against different antibiotics was similar to $E$. faecalis with the very high resistance to ampicillin in seven $(87.5 \%)$ cases, three $(37.5 \%)$ to piperacillintazobactam, five $(62.5 \%)$ to each of the ceftriaxone and cefotaxime. There was no case of vancomycin resistance detected with E. faecium. E. faecalis or E. faecium strains showed no resistance against linezolid (Table-II).

The vancomycin-resistant strains of $E$. faecalis were found to be positive for two van genotypes.
In the molecular analysis, vanA genotype was detected in one $(25 \%)$ isolate while vanB genotype was detected in three (75\%) cases. None of the other genotypes detected in any of the isolates (Table-III).

\section{DISCUSSION}

Enterococci constituted the healthy flora of gut of human and many animals, but now they have become an evident reason for nosocomial urinary infections and skin infections. Enterococci have been identified as a second known cause of nosocomial infections in US hospitals. ${ }^{17}$ The current study recorded a significant occurrence of enterococci in urine, pus and wound swabs. The number of

Table-II: Antibiogram of enterococci against various antibacterial drugs.

\begin{tabular}{lcc}
\hline $\begin{array}{l}\text { Name of } \\
\text { Antibiotic }\end{array}$ & $\begin{array}{c}\text { E. faecalis } \\
(n=139) n(\%)\end{array}$ & $\begin{array}{c}\text { E. faecium } \\
(n=8) n(\%)\end{array}$ \\
\hline Penicillin & $139(100)$ & $8(100)$ \\
Ampicillin & $109(78.4)$ & $7(87.5)$ \\
Ceftriaxone & $99(71.2)$ & $5(62.5)$ \\
Piperacillin-tazobactam & $98(70.5)$ & $3(37.5)$ \\
Cefuroxime & $90(64.7)$ & $5(62.5)$ \\
Ciprofloxacin & $75(54)$ & $4(50)$ \\
Clindamycin & $40(28.8)$ & $2(25)$ \\
Erythromycin & $37(26.6)$ & 0 \\
Vancomycin & $4(2.9)$ & 0 \\
Linezolid & 0 & 0 \\
\hline
\end{tabular}


Table-III: Distribution of van genotypes among VRE $(\mathrm{n}=4)$.

\begin{tabular}{lcc}
\hline Genotypes & $\begin{array}{c}\text { E. faecalis } \\
n(\%)\end{array}$ & $\begin{array}{c}\text { E. faecium } \\
n(\%)\end{array}$ \\
\hline vanA & $1(25)$ & 0 \\
vanB & $3(75)$ & 0 \\
vanC1 & 0 & 0 \\
vanC2/C3 & 0 & 0 \\
\hline
\end{tabular}

E. faecalis $(94.6 \%)$ was higher than the E. faecium (5.4\%). A different prevalence of E. faecalis $(62.5 \%)$ and E. faecium $(23.9 \%)$ and other enterococci $(13.6 \%)$ has also been reported. ${ }^{18}$ A study from Pakistan reported urine samples as a major source for the isolation of enterococci. ${ }^{14}$ The higher occurrence of enterococci had been reported as $43 \%$ from pus and $31 \%$ from the urine specimens with the distribution of $76 \%$ E. faecalis and $24 \%$ E. faecium which is slightly higher than the current study. ${ }^{4}$ The nosocomial enterococcal infections frequently tend to occur in surgical wounds and urinary tract of the patients. E. faecalis $(85.7 \%)$ found to be the major organism followed by the second prevalent organism $E$. faecium $(14.3 \%) .{ }^{17}$ The isolation of enterococci has also been reported other than these two sources, which predominantly include stool, sputum and throat swabs. E. faecalis (92\%) remained to be higher in frequency than the E. faecium (8\%), and these findings are close to the current study. ${ }^{19}$

The majority of the cases were between the age of $21-40(40.8 \%)$ and $41-60(39.5 \%)$ years and the enterococcal strains were isolated significantly higher from female patients. The occurrence of enterococci has been reported higher in female patients with a higher infection rate in $21-40$ years. ${ }^{19}$ The greater occurrence of enterococci in 50-60 years of age has been reported by another study. ${ }^{20}$

Enterococci have become resistant to most of the $\beta$-lactam antibiotics, aminoglycosides, and glycopeptide (vancomycin), which made them an important nosocomial pathogen. In this study, enterococci found to be highly resistant to penicillin, cephalosporin and other classes of antibiotics with the emergence of $2.9 \%$ as VRE. The only choice remained to treat the VRE in this study was linezolid. A higher number of VRE $(16.1 \%)$ with a similar resistance profile have been reported from a tertiary care paediatric hospital of Pakistan and linezolid remained the last option for the treatment of VRE. ${ }^{14}$ In an Indian study, the VRE found to be prevalent in $36 \%$ of the cases with the variable resistance to the different classes of antibiotics. The resistance against the ciprofloxacin
(58\%), piperacillin (54\%), tetracycline $(47 \%)$ and amoxicillin $(47 \%)$ remained high. Linezolid and tigecycline were reported as the treatment of choice to treat VRE infections. ${ }^{19}$ The difference in the vancomycin resistance with other studies could be due to the walk-in cases in the current study. The hospitalised patients are more susceptible to the infections by the vancomycin-resistant strains as a result of nosocomial infections. The injudicious use of cephalosporin, ticarcillin, piperacillintazobactam and even vancomycin antibiotics helps in the emergence of VRE. These antibiotics reach to a higher gastrointestinal concentration with less activity against the enterococci. ${ }^{21}$

The van genotype responsible for the emergence of VRE in the current study was vanB $(75 \%)$ and vanA $(25 \%)$ which were detected in E. faecalis strains. The predominant genotype responsible for the appearance of resistance against vancomycin was found to be vanA followed by vanC. ${ }^{18}$ VRE have most commonly van $A$ and van $B$ genotype, but rarely an unusual genotype vanM can be detected..$^{22}$ A similar prevalence of vanA and vanB among the VRE have been reported by another study using rapid PCR test. ${ }^{23}$ These results support the findings of the current study where the existence of only van $B$ and van $A$ found in the region. The existence of van $A$ has been reported in $>95 \%$ cases in some studies while a study also reported $3 \%$ of cases of van C1 genotype. ${ }^{24,25}$

Limitations of the study: Due to limited resources, detection of other van genotypes could not be performed. The disease outcome and clinical data could not be managed for walk-in patients.

\section{CONCLUSION}

E. faecalis and E. faecium constituted as the predominant strains among enterococci which are responsible for the nosocomial multidrug-resistant infection. A shift in the pattern of antibiotic resistance in enterococci has been observed, which is mediated by self-transferable plasmids with a broader host range. The emerging strains of VRE in the current study are a potential menace for therapeutic failure, which left the physicians with only linezolid as a therapeutic option. This study reports the emerging van $B$ and van $A$ multidrug-resistant genotype, which is worrisome. Prompt action is required to regulate the consumptions of antibiotics (particularly vancomycin), and implementation of effective infection control strategies, surveillance, screening procedures and preventive measures should be practised worldwide. 
Acknowledgements: Special thanks to the Laboratory Administration of Alrazi Health Care Lahore, Ms. Sonia Younas and Dr. Kashaf Junaid for their support.

\section{Conflict of Interest: None.}

\section{Grant Support \& Financial Disclosures: None.}

\section{REFERENCES}

1. Moreno MF, Sarantinopoulos P, Tsakalidou E, De Vuyst L. The role and application of enterococci in food and health. Int J Food Microbiol. 2006;106(1):1-24 doi: 10.1016/j.ijfoodmicro.2005.06.026

2. Arias CA, Murray BE. Emergence and management of drug-resistant enterococcal infections. Expert Rev Anti Infect Ther. 2008;6(5):637-655. doi: 10.1586/14787210.6.5.637

3. Kajihara T, Nakamura S, Iwanaga N, Oshima K, Takazono $\mathrm{T}$, Miyazaki T, et al. Clinical characteristics and risk factors of enterococcal infections in Nagasaki, Japan: a retrospective study. BMC Infect Dis. 2015;15:426. doi: 10.1186/s12879015-1175-6

4. Sreeja S, Sreenivasa Babu PR, Prathab AG. The prevalence and the characterization of the enterococcus species from various clinical samples in a tertiary care hospital J Clin Diagn Res. 2012;6(9):1486-1488. doi: 10.7860/ JCDR/2012/4560.2539

5. Amin H, Zafar A, Ejaz H, Jameel NU. Phenotypic characterization of ESBL producing Enterobacter cloacae among children. Pak J Med Sci. 2013;29(1):144-147. doi: 10.12669/pjms.291.2385

6. Nosheen S, Ejaz H, Zafar A, Ikram H. Antibacterial activity of penicillins alone and in combination with different agents against Staphylococcus aureus. Pak J Pharm Sci. 2017;30(2):393-397.

7. Jada S, Jayakumar K. Prevalence of Enterococcus species from various clinical specimens in Shri Sathya Sai Medical College and Research institute with special reference to speciation \& their resistance to vancomycin. Int J Med Clin Res. 2012;3(4):154-160. doi: 10.9735/0976-5530.3.4.154-160

8. Fisher K, Phillips C. The ecology, epidemiology and virulence of Enterococcus. Microbiology. 2009;155(6):17491757. doi: 10.1099 /mic.0.026385-0.

9. Mundy LM, Sahm DF, Gilmore M. Relationships between enterococcal virulence and antimicrobial resistance. Clin Microbiol Rev. 2000;13(4):513-522. doi: 10.1128/ CMR.13.4.513

10. Schouten MA, Hoogkamp-Korstaje JA, Meis JF, Voss A. Prevalence of vancomycin resistant enterococci in Europe. Eur J Clni Microbiol Infect Dis. 2000;19(11):816-822

11. Xu X, Lin D, Yan G, Ye X, Wu S, Guo Y, et al. vanM, a new glycopeptide resistance gene cluster found in Enterococcus faecium. Antimicrob Agents Chemother. 2010;54(11):46434647. doi: 10.1128/ AAC.01710-09

12. Batistao DW, Gontijo-Filho PP, Conceicao N, Oliveira AG, Ribas RM. Risk factors for vancomycin-resistant enterococci colonisation in critically ill patients. Mem Inst Oswaldo Cruz. 2012;107(1):57-63.
13. Zhou $X$, Arends JP, Kampinga GA, Ahmad HM, Dijkhuizen B, Van Barneveld P, et al. Evaluation of the Xpert vanA/vanB assay using enriched inoculated broths for direct detection of vanB vancomycin-resistant enterococci. J Clin Microbiol. 2014;52(12):4293-4297. doi: 10.1128/JCM.01125-14

14. Shaheen AS, Mehdi NA, Zafar AI, Zubair M, Javed H, Kabeer S, Abbas S, Ejaz H. Emergence of vancomycin resistant enterococci in paediatric patients. Pak J Med Health Sci. 2014;8(3):572-575.

15. Clinical and Laboratory Standards Institute (CLSI). Performance Standards for Antimicrobial Susceptibility Testing; $26^{\text {th }}$ ed. CLSI document M100S: CLSI, 2016.

16. Kariyama R, Mitsuhata R, Chow JW, Clewell DB, Kumon $\mathrm{H}$. Simple and reliable multiplex PCR assay for surveillance isolates of vancomycin-resistant enterococci. J Clin Microbiol. 2000;38(8):3092-3095.

17. Olawale KO, Fadiora SO, Taiwo SS. Prevalence of hospital acquired enterococci infections in two primary-care hospitals in Osogbo, Southwestern Nigeria. Afr J Infect Dis. 2011;5(2):40-46.

18. Nasaj M, Mousavi SM, Hosseini SM, Arabestani MR. Prevalence of virulence factors and vancomycin-resistant genes among Enterococcus faecalis and E. faecium isolated from clinical specimens. Iran J Public Health. 2016;45(6):806-813.

19. Srivastava P, Mehta R, Nirwan P, Sharma M, Dahiya SS. Prevalence and antimicrobial susceptibility of Enterococcus species isolated from different clinical samples in a Tertiary Care Hospital of North India. Natl J Med Res. 2013;3(4):389-391.

20. Barros M, Martinelli R, Rocha H. Enterococcal urinary tract infections in a university hospital: Clinical studies. Braz J Infect Dis. 2009;13(4):294-296. doi: 10.1590/S141386702009000400011

21. Rice LB. Emergence of vancomycin-resistant enterococci. Emerg Infect Dis. 2001;7(2):183-187.

22. Teo JW, Krishnan P, Jureen R, Lin RT. Detection of an unusual van genotype in a vancomycin-resistant Enterococcus faecium hospital isolate. J Clin Microbiol. 2011;49(12):4297-4298. doi: 10.1128/JCM.05524-11

23. Birgand G, Ruimy R, Schwarzinger M, Lolom I, Bendjelloul G, Houhou N, et al. Rapid detection of glycopeptideresistant enterococci: impact on decision-making and costs. Antimicrob Resist Infect Control. 2013;2(1):30. doi: 10.1186/2047-2994-2-30

24. Ibrahim RB, Mohamad M, Rahman MM. Vancomycin resistant enterococci and detection of responsible genes. Pak J Med Sci. 2011;27(4):784-788.

25. Praharaj I, Sujatha S, Parija SC. Phenotypic \& genotypic characterization of vancomycin resistant Enterococcus isolates from clinical specimens. Ind $\mathrm{J}$ Med Res. 2013;138(4):549-546. 